\title{
A novel assay combined PCR with junction probe for rapid detection of anaerobes DNA
}

\author{
Shuai Yan ${ }^{1}$, Li Zeng ${ }^{1 *}$, Wenlin $\mathrm{Li}^{1}$ and Haihua Qian ${ }^{2}$ \\ ${ }^{1}$ Nanjing University of Chinese Medicine, Nanjing, Jiangsu, ZIP: 210000. People's Republic of China. \\ ${ }^{2}$ Jiangsu Province Hospital of TCM, Nanjing, Jiangsu, ZIP: 210000. People's Republic of China.
}

Accepted 10 May, 2013

\begin{abstract}
Herein, we developed a novel assay for detection of anaerobes by polymerase chain reaction (PCR) combined with JP (junction probe). The PCR products were processed by bead-based extraction to get single strand DNA which could be used in the junction probe reaction. The experiment results demonstrate that the new assay which we call PCR-JP could detect at least 10 copies of DNA with high sensitivity and specificity which is easily operated in the elementary medical organizations and resource-limited areas.
\end{abstract}

Key words: Junction probe, PCR, Anaerobes, rapid detection.

\section{INTRODUCTION}

Anaerobes are prominent human pathogens and a leading cause of bacterial infection in hospitals (for example: necrotizing fasciitis) and the community. The treatment of infections caused by antibiotic-resistant bacteria is especially methicillin-resistant. Necrotizing fasciitis is a surgical emergency. Thus, routine studies in patients with necrotizing fasciitis may be challenging to interpret (Malghem et al., 2012) (Lebel, 2012). Anaerobes are bound to generate grievous consequences in Necrotizing fasciitis and community settings (Hazelton et al., 2013). Therefore, a rapid, specific, and sensitive detection method is in high demand for the early diagnosis and clinical therapy of Anaerobes. At present, the most extensively used detection method for anaerobes in developing countries still is the traditional bacterial culture, and this often needs a long culture period, which is disadvantageous to the treatment for patients (Marathe et al., 2012). To resolve this problem, newly developed diagnostic techniques such as real-time PCR have been widely used in developed countries, which is rapid, specific and sensitive for anaerobes diagnosis. However, the highly cost for real-time PCR equipment has limited the usage of this method in developing countries. Here, we presented a novel molecular assay that combined PCR with junction probe (JP) (Shizuka Nakayama et al., 2008) (Yan et al., 2010). The novel assay just needs a general PCR amplifier instrument and a simple fluorescence detector which is much more accessible for primary hospital in terms of price. The JP technology is an isothermal signal amplify assay which utilizes a strategy called TeHyP (an acronym for template enhanced hybridization processes). For the TeHyP strategy, two probes which have limited regions of complementarity do not form a duplex at ambient temperature but can be made to hybridize to each other in the presence of an analyte to form a tripartite. If the region of complementarity between the two probes $A$ and $B$ contains a restriction endonuclease sequence, then the formation of the tripartite structure will lead to the generation of a cognate site that is cleaved by a REase to form an unstable tripartite structure. Then, the fluorescence was released as the tripartite dissociated. In JP reaction, the same target could be used repeatedly to cleave multiple copies of probes, which generates a large amount of fluorescence signal. 
A fluorescence dictator was used in this research in order to confirm the detection results (Yan et al., 2010).

However, the JP assay was only available for detecting single strand DNA or RNA, so we cannot detect the PCR products directly by JP. To resolve this issue, we decided to utilize a biotinylated single-stranded template bound to magnetic microspheres to get single strand PCR products (Tang et al., 2006). The template was generated by a PCR-reaction with one of the two primers biotinylated. Then the biotinylated PCR-products were bound to streptavidin-coated magnetic beads. The non-biotinylated strands were removed using alkaline treatment and magnetic separation. Finally, the purified single strand DNA could be used in JP reaction.

\section{MATERIALS AND METHODS}

\section{Bacterial strands and DNA extraction}

Two bacterial strands were used in this research, and the clinical isolates of anaerobes was got from Jiangsu Province Hospital of TCM, the Mycobacterium tuberculosis which used as PCR negative control was previously stored in our lab. The bacteria were heated at $90^{\circ} \mathrm{C}$ for $10 \mathrm{~min}$, and then we extracted DNA by the TaKaRa MiniBEST bacterial genomic DNA extraction kit (Takara Bio. Co) in accordance with the manufacturer's instructions.

\section{Design of the PCR primers and JP probe}

Anaerobes epidermidis mecA gene (Genbank number: FJ810876.1) was chosen as the PCR target according to the previously published paper (Vremeră et al., 2011). The primers sequences were as follows: up-stream primer: 5'ATTCGACTGCACTCGACGG-3', down-stream primer: 5'ACCGATTGTACGTAGGACGTAC-3'. The PCR primers were designed by Gene Tool software and the up-stream primer was biotinylated for single strand extraction. The products fragment length was $62 \mathrm{bp}$. The JP probes were designed according to the biotinylated single strand PCR products followed the instruction of the previous article (Yan et al., 2010) (Figure 1).

\section{PCR reaction and single strand extraction}

The anaerobes DNA of initial concentration $10^{4}$ copies $/ \mu$ l was $10 \times$ serially diluted and $10^{3}, 10^{2}, 10^{1}$ and 1 copy were used respectively as the template to carry out the PCR reaction. M. tuberculosis was set up as a negative control. The conditions for the PCR were as follows: denaturation at $94^{\circ} \mathrm{C}$ for $1 \mathrm{~min}$, annealing at $60^{\circ} \mathrm{C}$ for $1 \mathrm{~min}$, and extension at $70^{\circ} \mathrm{C}$ for $90 \mathrm{~s}$, totally for 30 cycles. The biotinylated PCR product (200 to $300 \mathrm{ng}$ ) was bound to $25 \mu \mathrm{l}$ of magnetic streptavidin beads by incubation in $6 \times$ SSC for $2 \mathrm{~min}$. Beads were washed once in $6 \times \mathrm{SSC}$ and the nonbiotinylated strand was removed by two cycles of denaturation in $100 \mu \mathrm{l}$ of $0.125 \mathrm{M} \mathrm{NaOH}$. After washing twice with $1 \times$ SSC, $0.1 \%$ SDS and twice with $\mathrm{H}_{2} \mathrm{O}$ the template was labeled by a standard random priming reaction (Random Primed DNA-labeling Kit, Boehringer Mannheim). The beads were then washed ( $1 \times$ SSC), and the DNA was denatured by heating at $95^{\circ} \mathrm{C}$ for $5 \mathrm{~min}$. The supernatant was recovered and used in the JP reaction. To confirm the effect of single strand extraction we compared the electrophoresis results of PCR products and single strand extractive.

\section{JP reaction and results analytic}

The component of JP reaction solution was as follows: probes 1 $\mu \mathrm{M}$, template $1 \mu \mathrm{M}$, CVIQI $0.0267 \mathrm{U} / \mu \mathrm{L}$; buffer: $20 \mathrm{mM}$ Tris-acetate, $50 \mathrm{Mm} \mathrm{KOAc}, 10 \mathrm{mM} \mathrm{Mg}(\mathrm{OAc})_{2}, 1 \mathrm{mM}$ DTT, bovine serum albumin $(100 \mu \mathrm{g} / \mathrm{mL})$. The total volume for each reaction was $50 \mu \mathrm{L}$. The reaction was processed in a fluorescence detector with heating function (Veritas ${ }^{\mathrm{TM}}$. Yuan ping hao. Bio. Co) in $37^{\circ} \mathrm{C}$ for $20 \mathrm{~min}$, during reaction we used the fluorescence detector to measure the fluorescence of these single strand DNA samples extracted from PCR solution. A synthesized single strand DNA template was used as a positive control for JP reaction. The negative control DNA had a similar sequence with the positive control except one base was changed so it could not be complemented with the JP probes.

\section{RESULT}

\section{Result of PCR and bead-based single strand extraction}

The electrophoresis results show that PCR detected the minimal value of 10 copies anaerobes DNA and no electrophoretic bands were shown in the $M$. tuberculosis (Figure 2). The electrophoresis results of single strand extractive were consistent with the PCR products (Figure 2).

\section{JP results}

During the JP reaction the fluorescence could be detected in real time by a fluorescence detector. Within the $1 \mathrm{st}$ $20 \mathrm{~min}$ of the reaction, the intensity of fluorescence increased linearly for the positive control and anaerobes PCR samples. As an indication of the specificity of the reaction, the negative control which had only one base difference with the positive control gave no increase of signal (Figure 3).

\section{DISCUSSION}

In this research we have set up a new molecular diagnosis assay which combined PCR with JP reaction. Different from the Taqman probe, JP was a "probe amplification" assay which the same target could be used repeatedly to cleave multiple copies of probes (Shizuka Nakayama et al., 2008); therefore, it is more sensitive than Taqman assay. As JP was only available for detecting single strand DNA or RNA, we successfully designed a beads-based single strand extraction method to adapt JP reaction. The new method, which we call here PCR$\mathrm{JP}$, has many advantages when used in the bacterial DNA detection. Firstly, the new assay has a comparable sensitivity with Realtime-PCR but it does not ask for expensive equipment, which makes it more available in the elementary medical organizations and resource-limited areas. In our research, we approved that the PCR-JP could detect the minimal value of 10 copies DNA, however it just need a general PCR amplifier device and a 


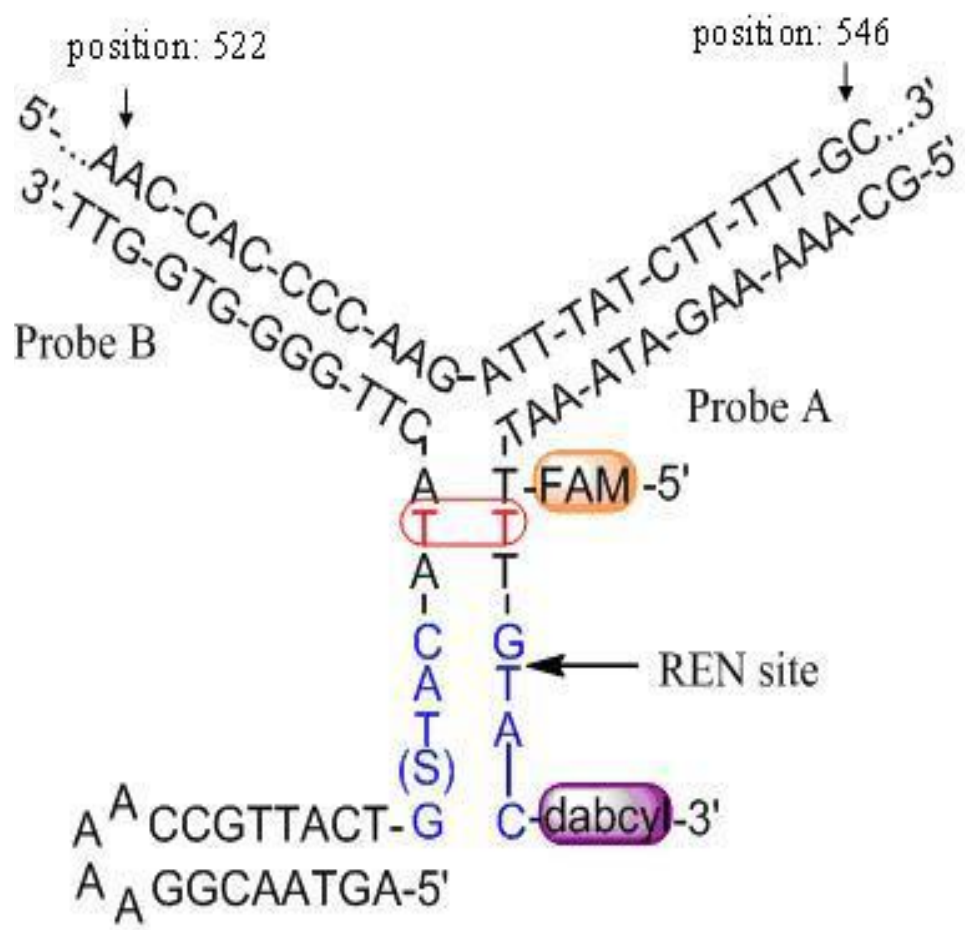

Figure 1. The structure of probe $A$ and probe $B$ for JP reaction. The target DNA sequence is at the top of the figure (position 522 to 546). Probe A is labeled with a fluorescent molecule (FAM) that is quenched by Dabsyl. The FAM and Dabsyl molecules are separated by a 7-mer sequence (5'TTTGTAC-3') that contains a CviQ I restriction endonuc-lease (REN) site. REN enzymes only cleave double-stranded DNA; therefore probe $A$ cannot be cleaved by CviQ I unless it hybridizes with a complementary strand to form a duplex. Probe A contains two regions that are complementary to part of a DNA/RNA target and probe B. Probe B is unlabeled and also contains two regions: a 7-mer region that is complementary to part of probe $A$ and a region that is complementary to part of a DNA/RNA target. The melting temperature of the duplex that results from probe $A$ annealing to probe $B$ is lower than $15^{\circ} \mathrm{C}$. Therefore, it was expected that in the absence of a template that can enhanced the hybridization of probes $A$ and $B$, CviQ I will be unable to cleave probes $A$ and $B$ because the two oligonucleotides will predominately exist as singlestranded at the reaction temperature $\left(30^{\circ} \mathrm{C}\right)$. However, in the presence of a DNA template, probes $A$ and $B$ and the template will hybridize to form a ternary " $Y$ " junction structure. One arm of the $Y$ junction structure contains a cleavage site for CviQ I and will be subsequently cleaved by this enzyme. The cleavage will then result in another ternary struc-ture which has a lower stability than the former complex, then, the Dabsyl will be separated so as to release the fluorescence of FAM. A modification of probe B with phosphorodithioate (5'-G(S) TAC-3') and a mismatch base pair (in red) was designed to enhance the cleavage rate.

fluorescence detector. Secondly, the specificity of the new assay was significantly improved by the combination with JP. As the probe of JP was designed according to the specific amplification products of PCR so even the reaction of PCR produced non-specific amplification products, JP would correct the mistake because the probe would not combine with the non-specific products and no fluorescence signal released. In our experiment, the negative template that only had one base changed from the positive template could be clearly distinguished by
JP, which was consistent with the previous research (Yan et al., 2010). Generally speaking, the combination of these two assays has improved both the specificity and sensitivity of the detection. Furthermore, in this new assay, the cycles of PCR can be reduced as JP was a signal amplification reaction. As we know that for PCR reaction, the fact is that the less cycles it process, the better specificity it has. Accordingly, next step we will focus on reducing the cycles of PCR in the PCR-JP assay so as to further improve the specificity of the reaction. 


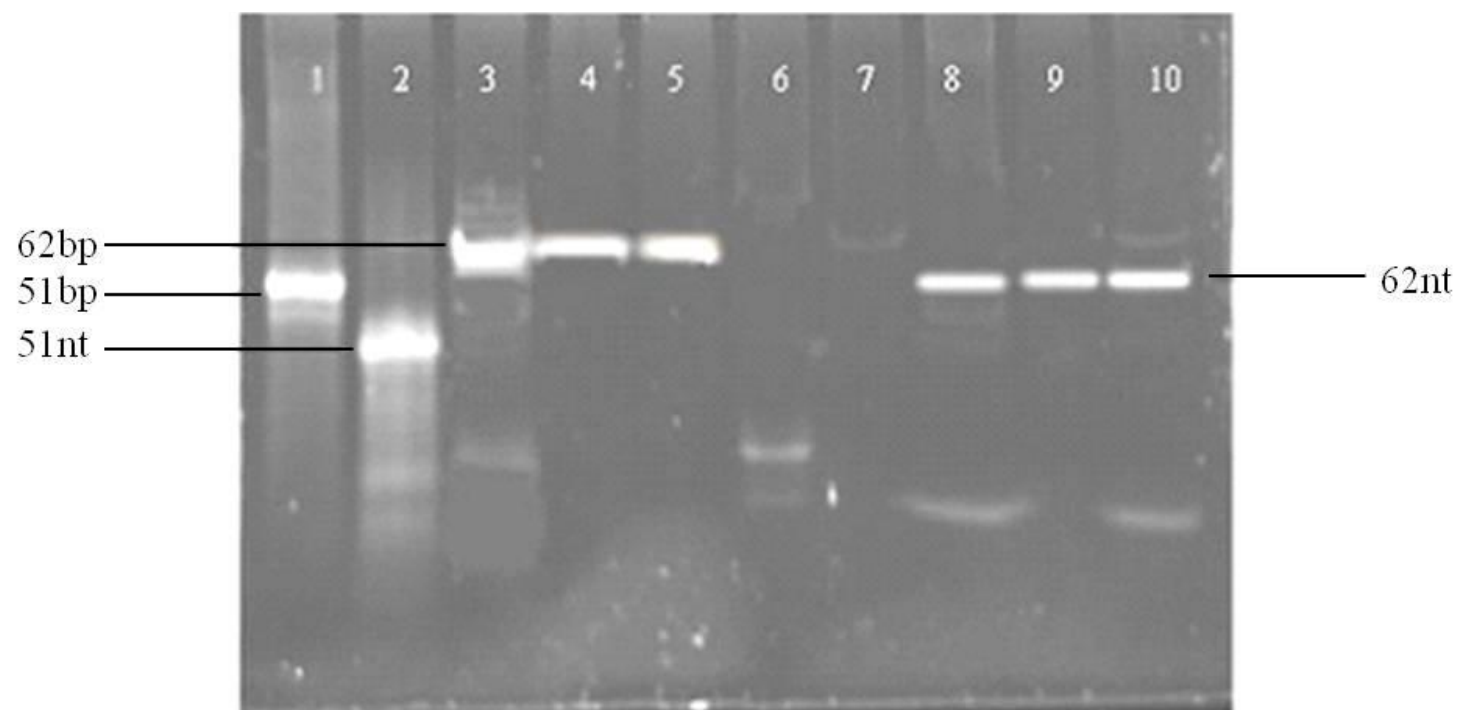

Figure 2. Electrophoresis results of PCR products and single strand extractive. Lane 1, DNA marker, $51 \mathrm{bp}$. Lane 2, single strand DNA marker, $51 \mathrm{nt}$. Lanes 3 to $7,10^{3}, 10^{2}, 10^{1}, 1$ copy of anaerobes DNA and the M. tuberculosis DNA. Lanes 8-10, single strand extractive from PCR products of samples 3 to 5.

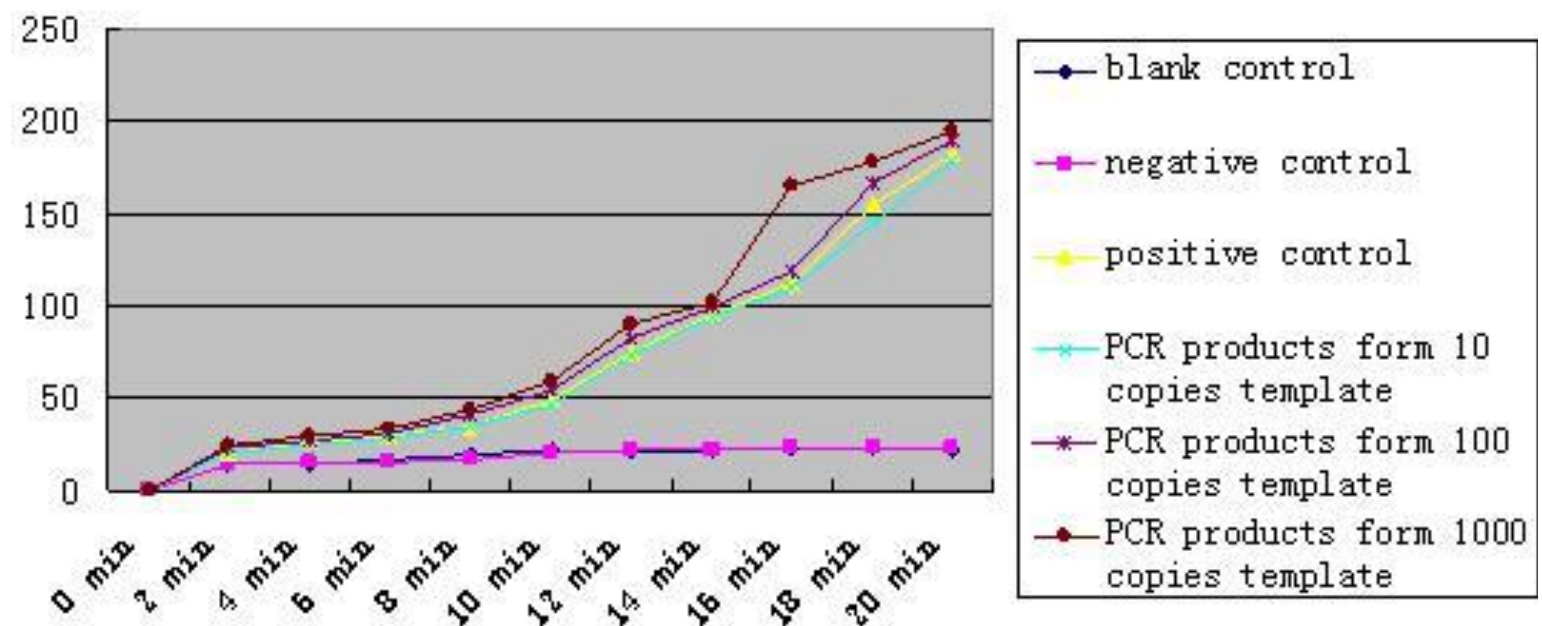

Figure 3. The fluorescence value of JP reaction in real time. The abscissa is reaction time; the ordinate is the fluorescence value. The intensity of fluorescence increased linearly for the positive control and single strand PCR products of Anaerobes samples.

\section{ACKNOWLEDGMENTS}

This work was financially supported by opening projects of TCM discipline of Nanjing University of Chinese Medicine (YS2012ZYX111). We are grateful to our teachers and colleagues who contributed to discuss this difficult topic in Nanjing University of Traditional Chinese Medicine for apparatus and technical assistance, particularly Ying Shao.

\section{REFERENCES}

Malghem J, Lecouvet FE, Omoumi P, Maldague BE, Vande Berg BC
(2012). Necrotizing fasciitis: Contribution and limitations of diagnostic imaging.Joint Bone spine. 53(6):1-9.

Lebel E, Karasik M, Shahroor-Karni S, Peyser A (2012). Necrotizing upper limb fasciitis in a newborn: an uncommon life-threatening event. J Pediatr Orthop B. 21(6):536-538.

Hazelton BJ, Thomas LC, Unver T, Iredell JR (2013). Rapid identification of Gram-positive pathogens and their resistance genes from positive blood culture broth using a multiplex tandem RT-PCR assay J MED MICROBIOL. 62(2):223-231.

Marathe N, Shetty S, Lanjekar V, Ranade D, Shouche Y (2012). Changes in human gut flora with age: an Indian familial study. BMC Microbiol.12:222.

Shizuka Nakayama, Lei Yan, and Herman O. Sintim (2008). Junction Probes - Sequence Specific Detection of Nuleic Acids via Template Enhanced Hybridization Processes. J. Am. Chem. Soc. 130(38):12560-12561. 
Tang X, Morris SL, Langone JJ, Bockstahler LE (2006). Simple and effective method for generating single-stranded DNA targets and probes. Biotechniques. 40(6):759-763.

Yadav MK, Kwon SK, Huh HJ, Chae SW, Song JJ (2011). Detection of methicillin-resistant Staphylococcus aureus (MRSA) from nasal samples by multiplex real-time PCR based on dual priming AT-rich primers. Folia Microbiol. 57(1):37-45.

Vremeră T, lancu LS, Logigan C, Năstase E, Miftode E, Luncă C,
Dorneanu O (2011). Optimization of triplex real time PCR for detecting Staphylococcus aureus mecA, pvl and nuc genes. Roum Arch Microbiol Immunol. 70(2):69-73.

Yan L, Nakayama S, Yitbarek S, Greenfield I, Sintim HO (2010). Isothermal detection of RNA with restriction endonucleases. Chem Commun (Camb). 47(1):200-2. 